pulmonary endarterectomy (PEA) surgery to remove the thromboembolic material. We also examined the surgically excised PEA material and measured neovessel density in distal regions of the specimens.

Methods and results Serum VEGF-A levels were measured by Luminex array in paired serum samples from $n=44$ patients at baseline (before PEA surgery) and following PEA surgery. Following PEA surgery, serum VEGF-A levels were significantly reduced compared to baseline measurements (159.5 \pm 174.8 vs. $194.4 \pm 198.2, \mathrm{p}=0.0182)$. Distal regions of excised PEA material were cross sectioned and processed for histopathological examination. Neovessel density was calculated by counting the absolute number of vascular channels present within a cross-sectional area and normalised to tissue area using Image J. We also measured the time between documented VTE and subsequent PEA surgery (VTE-PEA time) in patients with a documented VTE ( $\mathrm{n}=40 / 44$ [90\%] patients), and identified an inverse correlation between neovessel density and VTE-PEA time.

Conclusions Extensive angiogenesis was evident in distal PEA material from patients with CTEPH. VEGF-A is known to be a key regulator of angiogenesis and serum levels were significantly reduced following PEA surgery. Interestingly, a significant inverse correlation between neovessel density and VTE-PEA time was observed indicating that in the early stages following VTE, extensive angiogenesis is evident within the thromboembolus. Taken together these data provide further evidence that angiogenesis is an important mechanism in the attempted resolution of a VTE.

\section{S38 THE BRD4 INHIBITOR, JQ1 DECREASES PROLIFERATION AND ARRESTS THE CELL CYCLE OF PULMONARY VASCULAR CELLS: IMPLICATIONS FOR PULMONARY} ARTERIAL HYPERTENSION

${ }^{1} \mathrm{~S}$ Mumby, ${ }^{2} \mathrm{~N}$ Gambaryan, ${ }^{2} \mathrm{~A}$ Adcock, ${ }^{1} \mathrm{~S} J$ Wort. ${ }^{1}$ Vascular Biology, National Heart and Lung Institute, Imperial College London, London, UK; ${ }^{2}$ Airway Disease, National Heart and Lung Institute, Imperial College London, London, UK

\subsection{6/thoraxinl-2014-206260.44}

Background Pulmonary arterial hypertension (PAH) is an incurable disease characterised by raised pulmonary resistance, resulting from vascular remodelling which leads to right heart failure and death. Recently NF-kB mediated inflammatory gene expression and vascular proliferation/remodelling have been shown to be involved in the pathogenesis of PAH. The expression of subsets of NF-kB-induced inflammatory genes is dependent upon the recruitment of the bromodomains and extra-terminal (BET) family of proteins to the transcriptional activation complex. We hypothesise that inhibition of BET proteins which bind acetylated lysine residues on histones and non-histone proteins will attenuate the hyperproliferative and proinflammatory phenotype of vascular cells.

Methods Primary human pulmonary vascular endothelial cells (P-EC) were serum starved for $24 \mathrm{~h}$ prior to treatment with the Brd4 mimic JQ1+ or JQ1- (inactive enantiomer) in complete (5\% FCS) media. P-EC cell proliferation was measured by BrdU incorporation and apoptosis was determined using caspase 3/7 activity. Cell cycle progression was determined by FACs analysis. mRNA levels of cell cycle genes and inflammatory cytokines were measured by RT-PCR. MTT assay was used to measure cell viability.

Results JQ1+ caused a significant $(\mathrm{p}<0.001)$ and concentration-dependent decrease in P-EC proliferation and an increase in caspase 3/7 activity compared to P-EC treated with JQ1- for 24 h. JQ1+ $(1 \mu \mathrm{M})$ significantly arrested the cell cycle of P-EC at the G1 phase. This was additionally evidenced by a decrease in the cell cycle genes CDK2, 4 and 6 mRNA levels and a significant increase in the mRNA of the cell cycle inhibitor CDKN1A (p21/cip1) at 4 h. Finally, JQ1+ significantly $(\mathrm{p}<0.01)$ inhibited the mRNA levels of the inflammatory cytokines IL-6 and 8 in P-EC compared to JQ1-.

Conclusion Inhibition of Brd4 with JQ1 decreases remodelling and inflammation in P-EC via a decrease in proliferation, cell cycle arrest and an increase in apoptosis. Further work is required but Brd4 inhibition may provide therapeutic drugs for the treatment of PAH.

\section{S39 THE ROLE OF SOLUBLE GUANYLATE CYCLASE STIMULATOR BAY 41-2272 ON REMODELLING PROCESSES RELEVANT TO THE PATHOGENESIS OF PULMONARY ARTERIAL HYPERTENSION}

D Shao, SJ Wort. Imperial College London, London, UK

\subsection{6/thoraxjnl-2014-206260.45}

Introduction and objectives Pulmonary arterial hypertension (PAH) is characterised by remodelling of small, muscular precapillary blood vessels. The subsequent rise in pulmonary vascular resistance leads to right ventricular failure and death. The aetiology of the remodelling process is largely unknown although defects in the bone morphogenetic protein receptor II (BMPR II) pathway are likely to be involved. Most of the therapies used thus far are aimed at pulmonary vasodilation. However it is unclear how much of the benefit seen with these medications is related to reverse remodelling. Riociguat is a "first in class" drug that stimulates soluble guanylate cyclase, with a consequent increase in cyclic GMP (and vasodilation). Riociguat has recently been shown to improve haemodynamics and exercise capacity in patients with idiopathic $\mathrm{PAH}$ and chronic thromboembolic PH (PATENT and CHEST). Here we sought to

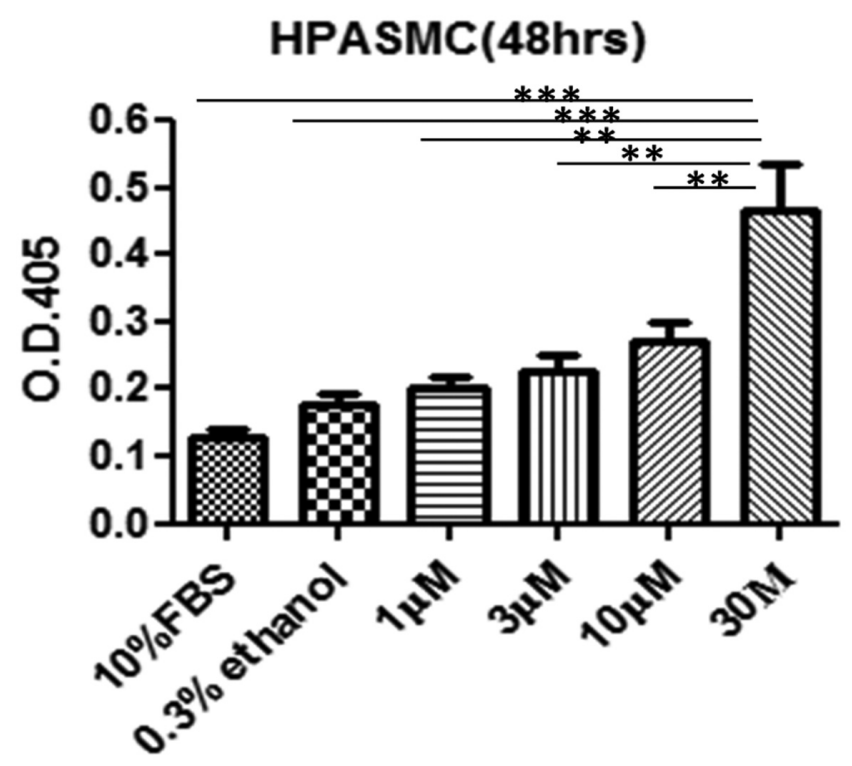

Abstract S39 Figure 1 Bay41-2272 induces HPASMCs DNA fragmentation (apoptosis), data were presented as mean \pm SEM $n=3$. ${ }^{* *} \mathrm{p}<0.005 ;{ }^{* *} \mathrm{p}<0.001$ 


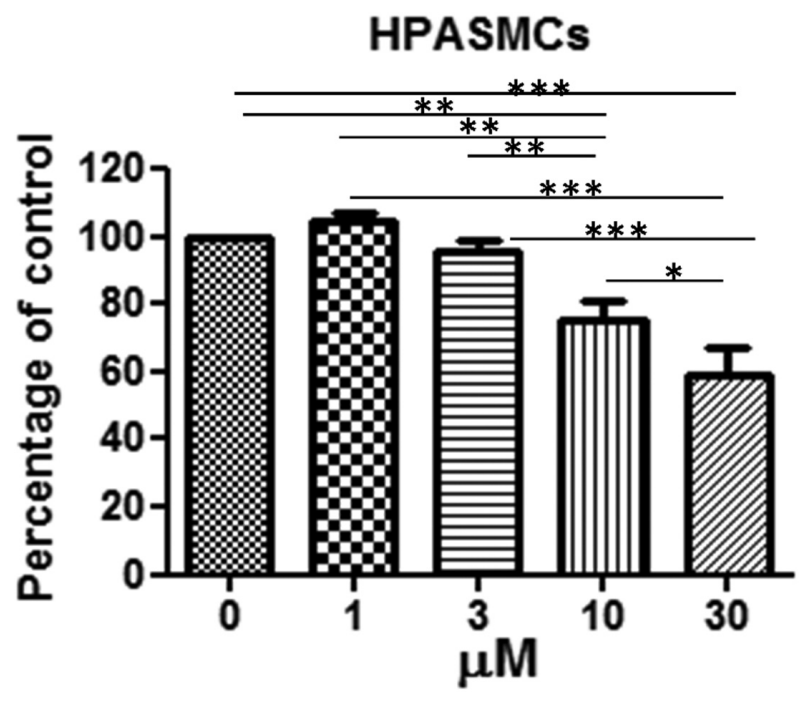

Abstract S39 Figure 2 Bay41-2272 inhibits HPASMC proliferation, data were presented as mean \pm SEM, $n=3$. ${ }^{*} p<0.05$; ${ }^{* *} p<0.005$; $* * * p<0.001$

determine the effects of Bay 41-2272, the tool compound for riociguat, on remodelling processes in pulmonary vascular cells. Methods We used primary human endothelial (HPAECs) and smooth muscle cells (HPASMCs) as our target cells. Proliferation was measured using the CyQUANT proliferation kit after cells were treated with various concentration of Bay 41-2272 (kind gifted by Bayer Pharmaceuticals Ltd) for $72 \mathrm{~h}$ in the presence of $15 \%$ serum. Apoptosis was measured using Cell death ELISA kit and DAPI staining after cells were treated with various concentration of Bay 41-2272 for 24 and $48 \mathrm{~h}$ in the absence of serum.

Results Bay41-2272 treatment increased HPASMC apoptosis after 24 and 48 h (Figure 1) by Cell death ELISA; this was further confirmed by DNA condensation assay (DAPI staining). Bay 41-2272 treatment also increased HPAEC apoptosis at $24 \mathrm{~h}$. It was not clear at $48 \mathrm{~h}$ treatment whether HPAEC cell death was significant in the absence of serum. Bay 41-2272 treatment reduced HPASMC proliferation (Figure 2), but had no effect on HPAECs.

Conclusions Our preliminary indicate that Bay 41-2272 increases apoptosis and inhibits proliferation, at least in HPASMCs, in vitro. Further studies are needed to fully characterise these effects on remodelling processes and to compare sGC stimulators, such as Bay 41-2272 to Type V phosphodiesterases.

\section{Images in pleural disease}

\begin{tabular}{l|l}
\hline S40 IMPROVING THE PATIENT JOURNEY: THORACIC \\
ULTRASONOGRAPHY AS AN ADJUNCT TO DECISION \\
MAKING AND DIAGNOSTIC PATHWAYS IN PLEURAL \\
DISEASE
\end{tabular}

JP Corcoran, RJ Hallifax, I Psallidas, A Talwar, A Sykes, NM Rahman. Oxford Centre for Respiratory Medicine, Oxford University Hospitals NHS Trust, Oxford, UK

\subsection{6/thoraxjn-2014-206260.46}

Background and method Pleural disease represents a growing source of referrals to respiratory services. Physicians increasingly provide many of the diagnostic and therapeutic interventions these patients require independent of colleagues in radiology or thoracic surgery. This changing practice can streamline diagnostic pathways within individual centres, and is reflected in BTS guidelines and the need for respiratory physicians to train in thoracic ultrasonography (TUS).

Patients referred to our tertiary-level service undergo in-depth TUS to help determine their diagnostic pathway; assessing factors including the nature of any pleural fluid, positioning of intercostal vessels, and movement of the underlying lung. We reviewed our procedural database (January 2010 to June 2014) and clinical records to identify cases where TUS influenced clinical decision making or subsequent investigations.

Results Procedural triage: 359 patients underwent assessment for diagnostic procedures to obtain pleural tissue during the study period. 64 patients were directed to have TUS-guided cutting needle pleural biopsies due to co-morbidity or after TUS identified heavily septated fluid and/or absent lung sliding (representative of adherent lung) that would prevent local anaesthetic thoracoscopy (LAT). One patient was referred for surgical biopsies after TUS identified septated fluid and an at-risk intercostal vessel that would prevent safe intervention by the physician team.

Advanced LAT: 294 LATs were scheduled during the study period. Four LATs were converted "on the table" to TUS-guided cutting needle biopsies after TUS identified increasing septation within the pleural space; a secure diagnosis was obtained in all cases.

95 LATs (32.3\%) required Boutin needle pneumothorax induction under TUS guidance. This was successful in 77 cases $(81.1 \%)$; in those LATs $(\mathrm{n}=18)$ where pneumothorax formation failed an attempt to obtain pleural tissue was made in 10 cases using TUS-guided cutting needle biopsies, making a secure diagnosis in 6 patients.

Conclusion TUS can greatly improve the patient's journey from presentation with pleural disease to diagnosis and should be utilised in all cases. TUS allows selection of the most appropriate means of obtaining diagnostic pleural tissue and facilitates more complex procedures. As interventional respiratory physicians become familiar with the capabilities of TUS this type of advanced practice may become increasingly widespread.

\section{S41 LOOKING BEYOND THE PLEURA - A SYSTEMATIC REVIEW OF THORACIC ULTRASONOGRAPHY TO DIAGNOSE LUNG CONSOLIDATION IN RESPIRATORY FAILURE}

${ }^{1} \mathrm{JP}$ Corcoran, ${ }^{2} \mathrm{PD}$ Wallbridge, ${ }^{1} \mathrm{NM}$ Rahman, ${ }^{3} \mathrm{~S}$ Mallett, ${ }^{4} \mathrm{M}$ Hew. ${ }^{1}$ Oxford Centre for Respiratory Medicine, Oxford University Hospitals NHS Trust, Oxford, UK; ${ }^{2}$ Department of Respiratory Medicine, Royal Melbourne Hospital, Melbourne, Australia; ${ }^{3}$ Nuffield Department of Primary Care Health Sciences, University of Oxford, Oxford, UK; ${ }^{4}$ Allergy, Immunology and Respiratory Medicine (AIRMED), The Alfred Hospital, Melbourne, Australia

\subsection{6/thoraxjnl-2014-206260.47}

Background and method The use of thoracic ultrasound (TUS) by physicians is increasingly commonplace in light of recent BTS guidelines and changes to training curricula. At its simplest, TUS enhances patient safety during interventions through the identification of pleural fluid and underlying structures. However, TUS training documents in the UK (Royal College of Radiologists) and US (American College of Chest Physicians) acknowledge a need for the ultrasonographer to recognise features of underlying lung, including consolidation. 